\title{
Perceptions des enseignants et des étudiants de première année licence SVT envers les caractéristiques l'enseignant et de l'enseignement
}

\section{Perceptions of teachers and first year students SVT towards teacher and teaching characteristics}

\author{
Said Mkhdramine ${ }^{1 *}$ and Khadija Essafi $^{1}$ \\ ${ }^{1}$ Laboratoire de didactique, innovation pédagogique et curruculaire, Faculté des Sciences Dhar El \\ Mehraz, Université Sidi Mohamed Ben Abdellah, Maroc.
}

\begin{abstract}
Résumé. Le but de cette étude descriptive concerne l'identification des caractéristiques de l'enseignant efficace et de l'enseignement efficace considérées comme très importantes de point de vue des enseignants et des étudiants de première année de la licence SVT. L'étude a été menée auprès de 41 enseignants et 290 étudiants, du département de Biologie de la Faculté de sciences Dhar El Mehraz. Ils ont été sollicités classer 60 items selon leur degré d'importance sur une échelle Likert. Les données issues des réponses au questionnaire montrent que les deux groupes participants expriment des points de vue similaires mais pas tout à fait identiques.
\end{abstract}

\begin{abstract}
The purpose of this descriptive study is to identify the characteristics of the effective teacher and effective teaching that are considered very important from the perspective of teachers and first- year SVT students. The study was conducted with 41 teachers and 290 students, from the Department of Biology at the Dhar El Mehraz Faculty of Science. They were asked to classify 60 items according to their degree of importance on a Likert scale. The data from the responses to the questionnaire show that the two participating groups express similar but not entirely identical points of view.
\end{abstract}

\section{Introduction}

L'augmentation massive des étudiants qui accèdent à l'université et la diversité de leurs profils, a débouché sur un constat d'échec et d'abandon le moins que l'on puisse dire préoccupant interrogeant les politiques sur la prise en charge de ces « nouveaux » étudiants. C'est ainsi que la réussite scolaire de ces étudiants, particulièrement ceux de la première

* Corresponding author: mkhdraminesaid@gmail.com 
année, continue de représenter un défi important pour les gestionnaires et les praticiens en éducation. [1] rapportent que depuis 1988 environ, le taux d'échec en première année universitaire a variéentre $66 \%$ et $75 \%$, alors que $52 \%$ des étudiants ne dépassaient pas le premier cycle universitaire et $60 \%$ abandonnaient avant la licence; $10 \%$ seulement obtenaient celle-ci en quatre années prévues dans l'ancien système. Toutefois, le rapport officiel du [31] critique à cet égard, nous rappelle d'une part, combien la question de la réussite est au cœur des préoccupations des responsables de l'enseignement supérieur. D'autre part, soulève de façon nouvelle et cruciale pour l'institution universitaire le problème des objectifs et de l'efficacité de la pratique pédagogique. Chose confirmée par le conseil supérieur de l'éducation (CSE), qui pointe du doigt une défaillance de nature pédagogique au supérieur: «... Au niveau de l'enseignement supérieur, et à cause du phénomène de la massification, les cours sont présentés de façon dogmatique, loin d'installer une pédagogie centrée sur l'étudiant»(p.67). Le conseil préconise ainsi, que la réforme doit viser «les pratiques pédagogiques et les méthodes d'enseignement »(p.100). En relation avec cette tendance, l'enjeu du présent article peut être situé au niveau du domaine qu'on appelle aujourd'hui « pédagogie universitaire ». Il vise d'identifier les caractéristiques à la fois de l'enseignant efficace et de l'enseignement efficace de point de vue des enseignants et des étudiants de première année au niveau du supérieur. A travers cette contribution nous envisageons de donner appui pour un renouveau pédagogique dans l'enseignement supérieur.

\section{Revue de littérature}

De nombreuses recherches ont tenté d'expliquer le phénomène de l'échec en première année de licence (L1) dans les études supérieures et d'identifier les facteurs liés à la réussite ou à la performance des étudiants. Cependant, la plupart des études qui se sont penchées sur l'amélioration de la réussite au niveau de la première année de Licence (L1), en vue d'entreprendre des actions efficaces de remédiation, se sont intéressées surtout aux déterminants inhérents à l'étudiant.

Parmi les principaux facteurs qui ont trait aux caractéristiques d'entrée de l'étudiant, misen évidence par les résultats de ces recherches, on cite :

* l'origine sociale [19, 14, 25].

* Le passé scolaire [14, 37, 19, 20, 32].

* Le projet personnel de l'étudiant [28, 27, 20).

* La maitrise de la langue française [12, 21]

* l'affiliation $[8,6]$

Malheureusement chacun de ces facteurs cités ci-dessus, s'est révélé par d'autres travaux $[26,37,5,18,10,11,38]$ peu influent, en précisant que d'autres facettes du phénomène ont été négligées.

Selon [34], l'étudiant est capable de surmonter toutes les carences individuelles à son entrée à l'université à condition qu'il soit soutenu « Tout n'était pas joué d'avance et qu'un étudiant très impliqué dans ses études pouvait compenser progressivement son "déficit »de départ, pour autant, bien sûr, qu'on lui en offre la possibilité» (p.26). Innocentant au moins partiellement l'étudiant, pour [15], d'autres dimensions concernant la problématique de l'échec n'ont pas encore été suffisamment explorées dans l'enseignement supérieur, comme l'écrit-elle :

«On admet plus ouvertement que le discours commun sur la baisse de niveau et l'inadéquation scolaire n'est plus tenable et que l'échec ne peut décemment être imputable aux seuls étudiants; on comprend enfin qu'il ne s'agit plus de trouver le dispositif qui permettra d'endiguer tous les échecs, mais qu'il s'agit bien d'adopter une approche plus " holistique », qui inclut, certes, des dispositifs d'accompagnement personnalisé mais suppose 
également des transformations en termes d'offre de formation, d'organisation pédagogique et de pédagogie... » (p.1)».

Dans le même ordre d'idées, [26] pointent du doigt l'institut universitaire et son fonctionnement qui n'offrent pas à l'étudiant un cadre favorable permettant de s'investir dans ses études «Si nombre d'entre eux (étudiants) n'ont pas reçu la formation suffisante pour suivre des études universitaires, le fonctionnement de l'université est un facteur aggravant qui vient se surajouter et accrô̂t le nombre d'échecs » (p.70), de ce fait Duru-Bellat (1995) nous prévient de ne pas négliger «les causes d'échec inhérents au fonctionnement même de l'université », étant donné qu'aucune recherche ne s'est jusqu'à présent véritablement intéressée au rôle joué par l'enseignant et sa pratique enseignante au supérieur [13].

C'est ainsi, que nous pouvons formuler l'hypothèse que le renforcement des compétences pédagogiques des enseignants et l'amélioration de leurs traits de personnalité constitueront probablement une autre orientation forte et une nouvelle piste de recherche. Surtout qu'il est reconnu que la qualité de l'enseignement et celle des enseignants, figurent parmi les déterminants de la satisfaction des étudiants dans l'enseignement supérieur [7].

De ce fait, le but recherché à travers ce travail, réside à déterminer les caractéristiques de l'enseignant efficace et de l'enseignement efficace évaluées comme très importantes de point de vue des enseignants et des étudiants de première année (L1). Bien sûr, il ne s'agit pas de dénoncer un trait, un comportement ou une pratique comme étant bonne ou mauvaise. Notre objectif est plutôt d'une part, d'avoir une idée sur les représentations que détient chaque groupe de répondants(enseignants et étudiants) du processus éducatif au supérieur. Il est d'autre part, de soulever les attentes et les souhaits d'une cohorte d'étudiants estimée comme très « fragile » à son entrée à l'université.

\section{Position du problème}

Le renouveau pédagogique est devenu une réalité imposée par les changements sans précédent que connait l'enseignement supérieur depuis près de 30 ans comme le montrent différents travaux de l'Unesco (réseau universitaire mondial pour l'innovation : GUNI) et de l'OCDE (programme IMHE : Institutional Management in Higher Education).

Les principales origines de ce renouveau sont :

- La massification de l'université : Les chercheurs qui se sont penchés sur la situation étudiante à partir du début des années 1990, soulèvent tous la brusque poussée des effectifs d'étudiants commencée dans les années 60, et qui se concentre surtout au niveau de la première année universitaire. Cette explosion démographique dans le nombre des étudiants s'est accompagnée de transformations qualitatives de leur population et a suscité chez eux et chez leurs enseignants, des «stratégies» et des pratiques dont les analyses classiques ne parvenaient plus à rendre compte. La massification de l'enseignement supérieur, selon [39], avait des répercussions négatives sur certaines dimensions de l'enseignement supérieur telles les curriculums, les formes d'enseignement, la relation recherche/enseignement et relation enseignement supérieur/enseignement secondaire. Pourtant la situation persiste pour longtemps " comme si rien ne devait changer ", malgré la dégradation à la fois de "la condition et le comportement des étudiants » [29]. Le Maroc ne fait pas exception, depuis les années quatre-vingt, le paysage de l'enseignement supérieur a beaucoup évolué. La grande masse des bacheliers s'est donc reportée sur l'université. Les publics accueillis sont maintenant plus larges. Dans un mouvement de popularisation de l'université, celle-ci n'est plus le lieu de production d'élite [22]. Selon [24], la massification est due à l'abolitiondu baccalauréat général fort sélectif au profit d'une formule semestrielle plus décentralisée (par académies) et beaucoup plus souple. Alors que d'autres tel [23], parlent même d'un " processus de dévaluation du baccalauréat » à la recherche de rendements exclusivement 
quantitatifs.

- Le fléau de l'échec : la difficulté de réussir la première année universitaire est signalée comme phénomène universel [30] et interpellant par son ampleur et son coût de plus en plus insupportable et économiquement intolérables [37]. [10] signalent que « Le phénomène de l'échec dans l'enseignement supérieur, et tout particulièrement en première année, interpelle à la fois par sa stabilité et par son ampleur. » (p.1).

Au Maroc, déjà en 2008, dans un rapport officiel, le Ministère de l'Education nationale a déclenché la sonnette d'alarme en mentionnant l'importante augmentation des échecs et des abandons d'étude, notamment chez les étudiants de première année. Dans les filières à accès ouvert, le rapport enregistre un taux de redoublement annuel moyen atteignant 30 pour cent. De sapart, Chami (cité par [20]) nous prévient que «Selon les statistiques de l'Enseignement supérieur, environ $50 \%$ des étudiants inscrits en première année quittent l'université au cours du premier cycle sans diplôme et la moyenne du nombre d'années d'études pour obtenir une licence est de 9.3 »(p. 65).

\section{Aspects méthodologiques}

\subsection{Public cible}

Ce travail vise l'identification des caractéristiques de l'enseignant efficace et de l'enseignement efficace perçues « très importantes » de point de vue des enseignants et des étudiants de première année licence SVT du département de Biologie.

L'échantillon est constitué de 41 enseignants (16 femmes : 39\%) et 290 étudiants (172 filles : $59,3 \%$ )

\subsection{Méthodologie}

Le questionnaire destiné à cette étude était réalisé à partir des résultats d'une enquête qualitative exploratoire menée auprès d'enseignants et d'étudiants de première année (L) du département SVT, qui visait l'identification des caractéristiques de l'enseignant efficace et de l'enseignement efficace de point de vue des deux groupes de répondants. Le questionnaire fut testé et rodé auprès de six étudiants et deux enseignants.

Par ailleurs, cette opération nous a permis l'obtention d'un questionnaire à questions fermées comportant 60 caractéristiques dont 30 désignant les qualités de l'enseignant efficace et 30 se rapportant à celles de l'enseignement efficace (voir Annexe). Il est à noter que les caractéristiques sont réparties de façon aléatoire. De plus, la version du questionnaire dédié aux enseignants est présentée en langue française, tandis que celle destinée aux étudiants est présentée en langue française avec une traduction en langue arabe pour ce qui a trait aux caractéristiques de l'enseignant efficace. En ce qui concerne les items se rapportant à l'enseignement efficace, ils ont été présentés uniquement en langue arabe, étant donné la longueur des énoncées, une insertion de la traduction en langue française à côté de la langue arabe surchargera le questionnaire. Les répondants (enseignants et étudiants) étaient sollicités à classer ces caractéristiques selon leur degré d'importance sur une échelle de Likert en quatre points : $1=$ Sans importance, $2=$ Peu important, $3=$ Important et $4=$ Très important.

Les données issues des perceptions des deux groupes de sondés ont été traités par SPSS (Statistical Packet for Social Sciences) version 20. En accord avec les méthodes employées par d'autres chercheurs dans le domaine, la variable « moyenne » est utilisée pour identifier le classement des différentes caractéristiques. Pour ce qui a trait aux limites réelles de chaque modalité, nous avons adopté celles qui ont été employées par [35] à savoir : 
1 - Sans importance (SI): les limites varient de 1,00 à 1,49

2 - Peu important (PI): les limites varient de 1,50 à 2,49

3 - Important (I): les limites varient de 2,50 à 3,49

4- Très important (TI): les limites varient 3,50 à 4,00 .

\section{Résultats}

Les réponses des enseignants et des étudiants au questionnaire ont permis de soulever leurs points de vue concernant le degré d'importance de chacune des différentes caractéristiques de l'enseignant efficace (première partie) et enseignement efficace (deuxième partie). Les résultats sont illustrés à travers les deux tableaux ci-dessous :

\subsection{Les caractéristiques de l'enseignant efficace perçues très importantes de point de vue desenseignants et des étudiants (L1)}

Les réponses des deux groupes de participants à la première partie du questionnaire constituée de 30 items, ont permis de connaître leur opinion quant aux qualités considérées très importantes (TI) pour désigner un enseignant efficace. Ainsi, comme affiché dans le tableau 4.1, huit caractéristiques selon les enseignants (bon communicateur (3.73), explique bien (3.68), assidu(3.63), ponctuel (3.63), expérience en enseignement (3.59), sérieux (3.54), patient (3.54), confiance en soi (3.51)) et douze caractéristiques selon les étudiants (Explique bien (3.92), dévoué (3.83), expert dans son domaine (3.76), respect ses étudiant (3.76), modèle pour les étudiants (3.76), bon communicateur (3.68), ponctuel (3.56), patient (3.56), expérience en enseignement (3.55), sérieux (3.55), assidu (3.52), bon écouteur (3.51) ont présenté une moyenne oscillant entre 4 et 3,5 .

Il semble si intéressant de mentionner que les enseignants ont évalué les 22 qualités restantes de l'instrument de l'enquête, comme importantes (I). Pour ce qui a trait aux étudiants, ils ont jugé 17 importantes (I) et seulement 2 comme étant peu importantes (PI). Ce qui montre que presque tous ces items s'avèrent essentielles pour décrire un enseignant efficace que ce soit pour les enseignants (moyenne de l'ensemble des qualités : 3,29) ou les étudiants (moyenne de l'ensemble des qualités : 3,21).

Le Tableau 4.1 ci-dessous montre les caractéristiques de l'enseignant efficace avec leur degré d'importance de point de vue des enseignants et des étudiants.

Tableau 1 : Degré d'importance des 30 caractéristiques de l'enseignant efficace selon les points de vue des enseignants et des étudiants (L1).

\begin{tabular}{|c|l|c|c|c|c|}
\cline { 3 - 6 } \multicolumn{2}{l|}{} & \multicolumn{2}{c|}{ Etudiants } & \multicolumn{2}{c|}{ Enseignants } \\
\hline $\begin{array}{l}\mathbf{N}^{\circ} \mathbf{d e} \\
\text { l'item }\end{array}$ & Caractéristiques & $\mathbf{M}$ & $\begin{array}{c}\text { D.I } \\
\cdot\end{array}$ & M & D.I. \\
\hline 23 & Explique bien & $\mathbf{3 , 9 2}$ & TI & $\mathbf{3 , 6 8}$ & TI \\
\hline 18 & $\begin{array}{l}\text { Dévoué dans son } \\
\text { travail }\end{array}$ & $\mathbf{3 , 8 3}$ & TI & $\mathbf{3 , 4 9}$ & I \\
\hline 12 & Respecte ses étudiants & $\mathbf{3 , 7 6}$ & TI & $\mathbf{3 , 4 1}$ & I \\
\hline 9 & $\begin{array}{l}\text { Expert dans son } \\
\text { domaine }\end{array}$ & $\mathbf{3 , 7 6}$ & TI & $\mathbf{3 , 4 9}$ & I \\
\hline 30 & $\begin{array}{l}\text { Modèle pour les } \\
\text { étudiants }\end{array}$ & $\mathbf{3 , 7 6}$ & TI & $\mathbf{3 , 3 7}$ & I \\
\hline 3 & Bon communicateur & $\mathbf{3 , 6 8}$ & TI & $\mathbf{3 , 7 3}$ & TI \\
\hline 1 & Patient & $\mathbf{3 , 5 6}$ & TI & $\mathbf{3 , 5 4}$ & TI \\
\hline 25 & Ponctuel & $\mathbf{3 , 5 6}$ & TI & $\mathbf{3 , 6 3}$ & TI \\
\hline
\end{tabular}




\begin{tabular}{|c|c|c|c|c|c|}
\hline 2 & Sérieux & 3,55 & TI & $\mathbf{3 , 5 4}$ & TI \\
\hline 20 & $\begin{array}{l}\text { Expérience en } \\
\text { enseignement }\end{array}$ & 3,55 & TI & 3,59 & $\mathrm{TI}$ \\
\hline 22 & Assidu & $\mathbf{3 , 5 2}$ & TI & 3,63 & $\mathrm{TI}$ \\
\hline 7 & Bon écouteur & 3,51 & TI & 3,46 & I \\
\hline 16 & Confiance en soi & 3,44 & $\mathrm{I}$ & 3,51 & $\mathrm{TI}$ \\
\hline 15 & Compréhensif & 3,38 & I & 3,24 & I \\
\hline 19 & Dynamique & $\mathbf{3 , 3 7}$ & I & 3,44 & $\mathrm{I}$ \\
\hline 24 & Instruit & 3,33 & I & 3,46 & I \\
\hline 29 & Poli & 3,31 & I & 3,32 & I \\
\hline 5 & Enthousiaste & 3,3 & I & 3,10 & I \\
\hline 21 & Modeste & 3,29 & I & 3,22 & I \\
\hline 4 & Accessible & 2,97 & I & 3,17 & I \\
\hline 10 & Gentil & 2,86 & I & 2,73 & $\mathrm{I}$ \\
\hline 27 & Une forte personnalité & 2,82 & I & 3,37 & $\mathrm{I}$ \\
\hline 6 & Volontaire & 2,74 & I & 3,12 & I \\
\hline 26 & Sympathique & 2,74 & $\mathrm{I}$ & 2,90 & $\mathrm{I}$ \\
\hline 13 & Utilise la technologie & 2,73 & I & 3,20 & I \\
\hline 14 & Flexible & 2,69 & $\mathrm{I}$ & 2,78 & I \\
\hline 28 & Souriant & 2,65 & $\mathrm{I}$ & 2,80 & $\mathrm{I}$ \\
\hline 17 & Approchable & 2,57 & I & 3,00 & I \\
\hline 8 & $\begin{array}{l}\text { Possède le sens de } \\
\text { l'humour }\end{array}$ & 2,25 & PI & 2,68 & I \\
\hline 11 & Bien présenté & 2,07 & PI & 2,95 & $\mathrm{I}$ \\
\hline & $\begin{array}{l}\text { Moyenne de } \\
\text { l'ensemble }\end{array}$ & 3,21 & & 3,29 & \\
\hline
\end{tabular}

M : Moyenne ; D.I : Degré d'importance ; TI : Très important ; I : Important.

\subsection{Les caractéristiques de l'enseignement efficace perçues très importantes de point de vue des enseignants et des étudiants (L1)}

Les résultats affichés dans le tableau 4.2 ci-dessous indiquent que les étudiants ont évalué 13 caractéristiques comme très importants (TI) pour décrire un enseignement efficace : la correction des examens se fait avec responsabilité (3.89), maîtrise de la matière à enseigner (3.84), l'évaluation doit être cohérente avec le contenu enseigné (3.81), le cours bien préparé et soigneusement expliqué (3.79), traitement des étudiants équitablement et sans discrimination (3.77), transmission des connaissances de manière facilitant la compréhension (3.72), l'enseignant est prêt à ré expliquer si c'est nécessaire (3.72), soutien et aide des étudiants en difficulté par l'enseignant (3.64), un environnement favorable à l'apprentissage (3.62), le contenudu cours doit être cohérent avec le contenu du TP (3.57), respect des étudiants et leur traitement avec sympathie (3.51), bonne attitude (impression) l'enseignant envers ses étudiants (3.51), amélioration continue de la pratique pédagogique (3.51).

De leur part, les enseignants ont évalué seulement 11 qualités comme très importantes à savoir : traitement des étudiants équitablement et sans discrimination $(3,85)$, la correction des examens se fait avec responsabilité $(3,80)$, transmission des connaissances de manière facilitant la compréhension $(3,78)$, maîtrise de la matière à enseigner $(3,78)$, le cours bien 
préparé et soigneusement expliqué $(3,76)$, l'évaluation doit être cohérente avec le contenu enseigné $(3,68)$, motivation des étudiants et stimulation de leur intérêt $(3,66)$, développement chez les étudiants le talent d'analyse et de réflexion $(3,61)$, développement chez les étudiants de l'esprit critique et de synthèse $(3,59)$, le contenu du cours doit être cohérent avec le contenu du TP $(3,56)$, l'enseignant est prêt à ré expliquer si c'est nécessaire $(3,51)$.

Tableau 2 : Degré d'importance des 30 caractéristiques de l'enseignement efficace selon lespoints de vue des enseignants et des étudiants (L1).

\begin{tabular}{|c|c|c|c|c|c|}
\hline \multirow[b]{2}{*}{$\begin{array}{l}\mathrm{N}^{\circ} \text { de } \\
\text { l'item }\end{array}$} & \multirow[b]{2}{*}{ Caractéristiques } & \multicolumn{2}{|c|}{ Etudiants } & \multicolumn{2}{|c|}{ Enseignants } \\
\hline & & $\mathbf{M}$ & D.I & $\mathbf{M}$ & D.I \\
\hline 17 & La correction des examens se fait avec responsabilité & 3,89 & TI & 3,80 & TI \\
\hline 2 & Maîtrise de la matière à enseigner & 3,84 & TI & 3,78 & TI \\
\hline 12 & $\begin{array}{l}\text { L'évaluation doit être cohérente avec le contenu } \\
\text { enseigné }\end{array}$ & 3,81 & TI & 3,68 & TI \\
\hline 14 & Le cours est bien préparé et soigneusement expliqué & 3,79 & TI & 3,76 & TI \\
\hline 20 & $\begin{array}{l}\text { Traitement des étudiants équitablement et sans } \\
\text { discrimination }\end{array}$ & 3,77 & TI & 3,85 & TI \\
\hline 3 & $\begin{array}{l}\text { Transmission des connaissances de manière facilitant la } \\
\text { compréhension }\end{array}$ & 3,72 & TI & 3,78 & TI \\
\hline 30 & L'enseignant est prêt à ré expliquer si c'est nécessaire & 3,72 & TI & $3, \mathbf{5 1}$ & TI \\
\hline 29 & $\begin{array}{l}\text { Soutien et aide des étudiants en difficulté par } \\
\text { l'enseignant }\end{array}$ & 3,64 & TI & 3,24 & I \\
\hline 26 & Un environnement favorable à l'apprentissage & 3,62 & TI & 3,34 & I \\
\hline 15 & $\begin{array}{l}\text { Le contenu du cours doit être cohérent avec le contenu } \\
\text { du TP }\end{array}$ & 3,57 & TI & 3,56 & TI \\
\hline 19 & Respect des étudiants et leur traitement avec sympathie & $\mathbf{3 , 5 1}$ & TI & 3,39 & I \\
\hline 21 & $\begin{array}{l}\text { Bonne attitude (impression) l'enseignant envers ses } \\
\text { étudiants }\end{array}$ & $3, \mathbf{5 1}$ & TI & 3,41 & I \\
\hline 18 & Amélioration continue de la pratique pédagogique & $3, \mathbf{5 1}$ & TI & 3,46 & I \\
\hline 4 & Motivation des étudiants et stimulation de leur intérêt & 3,47 & I & 3,66 & TI \\
\hline 23 & $\begin{array}{l}\text { Disponibilité de l'enseignant pour toute consultation } \\
\text { estudiantine }\end{array}$ & 3,45 & I & 3,37 & I \\
\hline 11 & $\begin{array}{l}\text { Encouragement des étudiants à participer aux } \\
\text { discussions et à échanger leurs idées }\end{array}$ & 3,39 & I & 3,39 & I \\
\hline 1 & Implication des étudiants dans le cours & 3,37 & I & 3,32 & $\mathrm{I}$ \\
\hline 25 & Initier les étudiants à la recherche & 3,36 & $\mathrm{I}$ & 3,20 & $\mathrm{I}$ \\
\hline 9 & Intégration de support facilitant l'apprentissage & $\mathbf{3 , 3 4}$ & I & 3,34 & I \\
\hline 5 & $\begin{array}{l}\text { L'enseignant prête attention aux besoins (académiques et } \\
\text { personnels) de ses étudiants }\end{array}$ & 3,32 & I & 3,44 & I \\
\hline 7 & $\begin{array}{l}\text { Développement chez les étudiants du talent de réflexion } \\
\text { et d'analyse }\end{array}$ & 3,26 & I & 3,61 & $\mathrm{TI}$ \\
\hline 27 & $\begin{array}{l}\text { Incitation des étudiants à être des explorateurs de } \\
\text { connaissances / auto-apprentissage }\end{array}$ & 3,17 & I & 3,34 & I \\
\hline 24 & $\begin{array}{l}\text { L'enseignant incite les étudiants à s'engager } \\
\text { sérieusement dans leurs études }\end{array}$ & 3,17 & I & 3,32 & I \\
\hline 28 & $\begin{array}{l}\text { Le contenu du cours permet une acquisition de } \\
\text { connaissances nouvelles et de valeur }\end{array}$ & 3,16 & I & 3,34 & I \\
\hline 10 & $\begin{array}{l}\text { Intégration de la technologie favorisant l'amélioration de } \\
\text { la qualité de l'enseignement }\end{array}$ & 3,13 & I & 3,27 & I \\
\hline
\end{tabular}




\begin{tabular}{|c|l|c|c|c|c|}
\hline 16 & $\begin{array}{l}\text { Le contenu du cours est adapté au progrès de la } \\
\text { recherche en la matière }\end{array}$ & $\mathbf{3 , 0 8}$ & I & $\mathbf{3 , 3 7}$ & I \\
\hline 8 & $\begin{array}{l}\text { Développement chez les étudiants de l'esprit critique et } \\
\text { de synthèse }\end{array}$ & $\mathbf{2 , 9 2}$ & I & $\mathbf{3 , 5 9}$ & TI \\
\hline 6 & Variation de méthodes d'enseignement & $\mathbf{2 , 9 0}$ & I & $\mathbf{2 , 9 3}$ & I \\
\hline 22 & $\begin{array}{l}\text { Intérêt et préoccupation de l'enseignant envers les } \\
\text { étudiants et leur réussite }\end{array}$ & $\mathbf{2 , 8 4}$ & I & $\mathbf{3 , 1 0}$ & I \\
\hline 13 & Pratique de contrôles continus & $\mathbf{2 , 5 9}$ & I & $\mathbf{3 , 2 9}$ & I \\
\hline \multicolumn{2}{|l|}{ Moyenne de l'ensemble } & $\mathbf{3 , 3 9}$ & & $\mathbf{3 , 4 4}$ & \\
\cline { 1 - 3 }
\end{tabular}

M : Moyenne ; D.I : Degré d'importance ; TI : Très important ; I : Important.

Il apparaît bien clair, d'après les données présentées dans le tableau 4.2, que les 30 items constituant la $2^{\text {ème }}$ partie du questionnaire et se rapportant aux caractéristiques de l'enseignement efficace ont été perçues comme très importantes ou importantes que ce soit par les enseignants (moyenne de l'ensemble des qualités : 3,44) ou les étudiants (moyenne de l'ensemble des qualités : 3,39 ). Nous supposons que de telles données témoignent de la pertinence de l'instrumentutilisé dans cette étude.

La première tendance massive qui peut être dégagée des résultats est le nombre prédominant de caractéristiques jugées très importantes par les étudiants par comparaison aux enseignants. Ainsi, les étudiants ont attribué la modalité «très important » à 12 qualités de l'enseignant efficace et 13 qualités de l'enseignement efficace, alors que les enseignants ne l'ont accordé qu'à 8 et 11 qualités respectivement. La deuxième tendance consiste au nombre, qu'on estime assez élevé, de qualités jugées comme très importantes, à la fois, par les deux groupes de répondants. On note 7 caractéristiques décrivant l'enseignant efficace ( explique bien, bon communicateur, patient, ponctuel, sérieux, a de l'expérience dans l'enseignement et assidu) et huit caractérisant l'enseignement efficace (La correction des examens se fait avec responsabilité, Maîtrise de la matière à enseigner, La cohérence entre l'évaluation et le cours enseigné, Le cours est bien préparé et soigneusement expliqué, Traitement des étudiants équitablement et sans discrimination, Transmission des connaissances d'une manière qui facilite la compréhension, L'enseignant est prêt à réexpliquer si c'est nécessaire, Cohérence entre le contenu du cours et le sujet du TP). Ces résultats sont en accord avec ceux rapportés par [16, 17, 35, 2] selon lesquelles, il existe une certaine similarité dans les perceptions des enseignants et des étudiants concernant les caractéristiques de l'enseignant/enseignement efficace, cependant, elles ne sont pas tout à fait identiques.

\section{Discussion}

L'analyse des données émergeantes de cette étude, nous permet de distinguer tout d'abord chez les étudiants, que les caractéristiques évaluées comme très importantes peuvent être réparties en trois groupes de caractéristiques se référant chacun à un type de problème d'affiliation.

- Problème d'affiliation académique : on y trouve comme caractéristiques : explique bien, dévoué, expert, l'enseignant est prêt à ré expliquer, soutien et aide des étudiants en difficulté par l'enseignant, le cours est bien préparé et soigneusement expliqué, un environnement favorable à l'apprentissage. En fait, chez les étudiants novices, on ressent tout le poids que peuvent revêtir, les difficultés d'une transition d'un système scolaire dont ils ont bien assimilé les règles et les lois à un autre méconnu pour eux et possédant ses allants de soi, ses us et coutumes. Ainsi, ces étudiants ont des difficultés à appréhender les nouvelles méthodes d'enseignement au supérieur, loin de leur expérience lycéenne, elles sont pour eux aussi surprenantes que déstabilisantes. Car, ces méthodes sont basées sur la transmission de 
savoirs savants dont ces néo- bacheliers en éprouvent des difficultés à s'approprier. D'après [3], les étudiants de première année souffrent des problèmes de prise de notes. En plus, cette transition secondaire- université constitue une source de remaniements plus ou moins importants de l'ensemble de leurs investissements scolaires, parce que le niveau acquit par ces derniers est insuffisant et le décalage entre les contenus d'enseignement du secondaire et du supérieur est trop grand, où les étudiants y voit trop de connaissances nouvelles [3]. A ce propos [9] rapportent que « Les étudiants, confrontés aux règles du savoir scientifique, sont contraintsd'entrer dans le jeu de la construction de nouvelles connaissances » (p.23). Ainsi, nous constatons que les étudiants novices donnent plus d'importance aux qualités qui se rapportent à la compréhension et l'assimilation du contenu disciplinaire.

Les données montrent aussi, que les «nouveaux » étudiants ne savent pas ce qu'on attend d'eux lors des évaluations. Il apparaît que lors de la prise de notes en cours magistral, ils ne saventpas effectuer un tri entre ce qui est essentiel et ce qui relève du secondaire, donc à s'approprier le contenu nécessaire et pertinent pour passer l'examen. En conséquence, ils n'arrivent pas à décrocher de bonnes notes, alors qu'ils avaient l'impression de connaître leurs cours. Ils pensent être victime d'une injuste correction et/ou les questions des examens ne reflètent pas le contenu vuen cours, comme le montre les degrés d'importance attribués à ces deux qualités : la correction des examens se fait avec responsabilité, l'évaluation doit être cohérente avec le contenu enseigné. Il semble que l'essentiel du cours leur échappe, ils n'arrivent pas à repérer les points importants.

- $\quad$ Problème d'affiliation linguistique : des caractéristiques telles : bon communicateur, expérience en enseignement, transmission des connaissances de manière facilitant la compréhension, reflètent en réalité une incapacité des étudiants à bien s'approprier les discours professoraux. L'entrée aux études supérieures constitue un hiatus, une rupture linguistique, étant donné que pendant toute la période pré-universitaire, les matières scientifiques étaient dispensées en langue arabe, cependant, au supérieur elles sont enseignées en langue française. Ainsi, disposant d'un faible niveau en français, ils ont énormément du mal à comprendre ces discours pédagogiques nouveaux et spécialisés communiqués en langue française, et par la même ils ont dumal à suivre la complexité d'une pensée scientifique, aussi bien dans un texte écrit que dans un exposé oral.

D'une manière générale, l'entrée à l'université confronte le nouvel étudiant à des problèmes la fois de connaissances et de méthodologie.

- $\quad$ Problème d'affiliation sociale : le groupe de caractéristique se rapportant à ce problème est composé des qualités suivantes : modèle pour les étudiants, patient, bon écouteur, respect des étudiants et leur traitement avec sympathie, bonne attitude (impression) l'enseignant envers ses étudiants, soutien et aide des étudiants en difficulté par l'enseignant, traitement des étudiants équitablement et sans discrimination. Ces caractéristiques montrent bien que les étudiants s'attendent de la part de leurs enseignants à de l'estime, du respect, de l'aide, du soutien, de la sympathie, afin de surmonter leurs difficultés et par la même faciliter leurs insertions scolaires. Ainsi, comme l'invoquent [9], une meilleure intégration des étudiants dansle monde universitaire passe certainement par une amélioration des relations dans l'enseignement supérieur, qui demeurent faibles. Dans le même ordre d'idées, [33] indique que les difficultés auxquelles font face les étudiants à l'université relèveraient davantage d'ordre relationnel que d'ordre pédagogique. En réalité, à cette période de leur âge, correspondant selon lathéorie du développement humain, à ce que [4] appelle l' " emerging adulthood » ou l'émergence de l'âge adulte, une étape de vie critique d'après les spécialistes. Les étudiants très sensibles s'attendent à ce qu'ils soient traités comme des «personnes » de la part de leurs enseignants et cherchent de la reconnaissance d'eux même et de leurs compétences. 
Pour ce qui a trait aux enseignants, nous constatons que malgré les nombreux facteurs contribuant à la dévalorisation de l'activité d'enseignement, tels l'alourdissement de la tâche du corps enseignant par la multitude de missions aussi générales que nombreuses, notamment des tâches administratives; l'absence de formation initiale et/ou continue en pédagogie; la liberté académique fondement même du statut universitaire, qui consiste à valoriser en premier lieu la spécialité de recherche par rapport à l'enseignement, cependant, par l'entremise des données affichées, nous découvrons en conformité avec les exigences de la réforme (objectivité et l'équité dans les évaluations et les examens, traitement des apprenants sur pied d'égalité, leur porter plus d'intérêt, leur être un modèle) une tentative constante d'investissement des enseignants dans la remédiation des difficultés que connaissent les étudiants à leur rentrée à l'université. Ils sont conscients motivations et de rapport aux études [37] et conscients également des problèmes tout d'abord qu'ils sont confrontés effectivement à de nouveaux profils d'étudiants, en matière de compétences, de et des contraintes auxquelles ces étudiants font face. D'après [36], les enseignants s'accordent sur le fait que « les étudiants de premier cycleont des difficultés de compréhension des connaissances dispensées dans les cours magistraux classiques, des problèmes méthodologiques de prise de notes et de travail personnel, d'appropriation de ces cours, sans compter un réel manque de relation enseignants/étudiants ». (p.42).

Ce qui fait voir à ces enseignants, sur le plan pédagogique, la nécessité d'adapter leurs interventions aux niveaux et aux besoins des étudiants favorisant une meilleure compréhension et assimilation des sujets présentés à ces derniers, comme le montre la nature des qualités jugées très importantes par ces derniers telles : explique bien, maîtrise de la matière à enseigner, le cours est bien préparé et soigneusement expliqué, transmission des connaissances de manière facilitant la compréhension, l'enseignant est prêt à ré expliquer si c'est nécessaire, motivation des étudiants etstimulation de leur intérêt.

Les enseignants montrent aussi de l'intérêt aux qualités qui favorisent le perfectionnement des habiletés mentales chez de leurs étudiants telles : développement chez les étudiants de l'esprit critique et de synthèse, développement chez les étudiants du talent de réflexion et d'analyse. Des aptitudes que doit développer chaque futur universitaire.

On ressent aussi, chez les enseignants enquêtés une large connaissance des difficultés auxquelles font face les étudiants de première année à déchiffrer les codes de l'évaluation à l'université. De ce fait, ils s'accordent à ce que l'évaluation porte sur ce qui a été vu dans le cours pendant le semestre, comme ils tiennent beaucoup à assurer les notions de justesse et de transparence dans la correction des examens concernant l'activité évaluative (la correction des examens se fait avec responsabilité, l'évaluation doit être cohérente avec le contenu enseigné).

De point de vue relationnel, les enseignants s'efforcent d'être plus équitables et flexibles dans le traitement avec les étudiants (traitement des étudiants équitablement et sans discrimination, patient, bon communicateur).

\section{Conclusion}

En guise de conclusion, il a été constaté que les étudiants de première année constituent une cohorte d'étudiants très fragile académiquement, linguistiquement et socialement. La plupart d'entre eux ne se sentent pas capables de répondre aux demandes de l'enseignement supérieur. Leurs perceptions quant à l'enseignant et l'enseignement efficaces se sont focalisées sur les qualités de relation enseignant-étudiant, la facilité de l'apprentissage du contenu disciplinaire et sur l'adéquation de l'évaluation. Les enseignants de leur côté, à 
travers toutes les caractéristiques qu'ils ont jugé très importantes, reconnaissent surtout les carences à la fois cognitives et linguistiques que connaissent les néo-bacheliers à leur entrée à l'université. En conséquence, ils s'efforcent d'apporter les remédiations adéquates à travers des qualités personnelles et des pratiques pédagogiques pertinentes.

\section{Bibliographie}

1. H. Akrim, S. El Jamali, A. El-Bousaadani, M. Radid \& M. Talbi, L'intégration des QCM dans le contrôle continu des étudiants de 1ère année universitaire SMP et SMC1. Cas de la Chimie Générale. RADISMA. Numéro, 1 (2006).

2. N.S. Al-Hinai, Effectuive college teaching and students rating of teachers: what students think, what faculty believe, and what actuel ratings show implications for policy and practice in a teaching quality assurance and control in higher education in Oman. These de doctorat, Université de Durham, (2011). Available at Durham E-Theses Online: http://etheses.dur.ac.uk/649/

3. M. Altet, Le cours magistral universitaire : un discours scientifico-pédagogique sans articulation enseignement-apprentissage. Recherche et Formation, 15, 9 (1994)

4. J.J. Arnett, Emerging Adulthood : A Theory of Development From the Late Teens Through the Twenties. American Psychologist, 55, 11 (2000)

5. S. Biémar, M. Philippe et M. Romainville, «L'injonction au projet: paradoxale et infondée? », L'orientation scolaire et professionnelle, 32, 20 (2003)

6. R. Boyer, C. Coridian \& V. Erlich, L'entrée dans la vie étudiante. Socialisation et apprentissages. Revue Française de Pédagogie, 136, 10 (2001)

7. A. Ceyhan et G. Yaprak, Student Satisfaction in Higher Education : a Turkish Case; Higher EducationManagement and Policy, 16, 13 (2004)

8. A. Coulon, Un instrument d'affiliation intellectuelle : l'enseignement de la méthodologie documentaire dans les premiers cycles universitaires. Bulletin de Bibliothèques de France, 44, 6 (1999)

9. A. Coulon \& S. Paivandi, Etat des savoirs sur les relations entre les étudiants, les enseignants et les IATOSS dans les établissements d'enseignement supérieur. Paris : Observatoire nationale de la Vie Etudiante (2008)

10. F. Crepin \& I. Demonty, L'amélioration de la transition entre l'enseignement secondaire et supérieur- présentation de projets pilotes associant des enseignants du secondaire et du supérieur en Communautéfrançaise de Belgique. Liège : Université de Liège (2009)

11. J.M. De Ketele, La pédagogie universitaire, un courant en plein développement. Revue Française de Pédagogie, 172, 8 (2010)

12. O. Dezutter et F. Thyrion, Comment les étudiants entrants s'approprient-ils les discours universitaires? Spirale - Revue de Recherches en Éducation, 29, 13 (2002)

13. A. Duguet et M. Morlaix, «Les pratiques pédagogiques des enseignants universitaires : Quellevariété pour quelle efficacité ? », Questions Vives [En ligne], 6, 7 (2012)

14. M. Duru-Bellet, « Des tentatives de prédiction aux écueils de la prévention en matière d'échec en première année d'université », Savoir Education Formation, 3, 17 (1995)

15. Endrizzi L.. Savoir enseigner dans le supérieur, un enjeu d'excellence pédagogique. Dossierd'actualité de l'INRP, 64, 23 (2011)

16. K. A. Feldman, The perceived instructional effectiveness of college teachers as related to their personality and attitudinal characteristics: a review and synthesis, Research in Higher Education, 24, 53 (1986) 
17. A. T. Fisher, J. G. Alder et M. W. Avasalu, Lecturing performance appraisal criteria: staff and student differences. Australian Journal of Education ,42, 15 (1998)

18. B. Galand, S. Neuville et M. Frenay, L'échec à l'université en Communauté Française de Belgique :Comprendre pour mieux prévenir ? Cahiers de Recherche en Education et Formation, 39, 12 (2005)

19. L. Gruel, Les conditions de réussite dans l'enseignement supérieur. OVE Infos, 2, 9 (2002)

20. N. Gury, Les sortants sans diplôme de l'enseignement supérieur : temporalités de l'abandon et profilsdes décrocheurs. Orientation scolaire et professionnelle, 36, 19 (2007)

21. M. Haidar, L'enseignement du français à l'université marocaine. Le cas de la filière « Sciences de la Vie et Sciences de la Terre et de l'Université ». Thèse de doctorat, Université Ibn Tofail et Université Rennes 2 (2012)

22. M. Kleiche, La Recherche Scientifique au Maroc, 1ère Partie : Histoire et institutions Paris : IRD, 35 (2002)

23. A. Lahlou, «L'École et l'Université à l'épreuve de la transformation sociale. Défis de l'éducation dans les pays émergents». Casablanca Edition Al madariss (2014)

24. L. Lahlou, La réforme de l'Université marocaine entre idéal organisationnel et réalité pratique. Colloque international organisé par l'université Paris VIII à l'occasion de son quarantième anniversaire 11-14 mai (2009)

25. M. Lambert-Le Mener, La performance académique des étudiants en première année universitaire :influence des capacités cognitives et de la motivation Thèse de doctorat. Université de Bourgogne, Dijon (2012)

26. D. Lapeyronnie et J. Marie. Campus bleus, les étudiants face à leurs études, édition Seuil. Paris (1992)

27. D. Lassarre, C. Giron, \& B. Paty, Stress des étudiants et réussite universitaire : les conditions économiques, pédagogiques et psychologiques du succès. Orientation scolaire et professionnelle, 32, 72 (2003)

28. S. Lemaire, Les facteurs de réussite dans les deux premières années d'enseignement supérieur(DEUG, DUT, BTS). Note d'information, 25, 5 (2000)

29. L. Lévy-Garboua, Les demandes de l'étudiant ou les contradictions de l'université de masse. RevueFrançaise de Sociologie, 17, 27 (1976)

30. D. Maurice, Réussir la première année à l'université. La transition Secondaire-Université : le projet Boussole. Revue française de pédagogie, 13, 9 (2001)

31. Ministère de l'Éducation Nationale, de l'Enseignement Supérieur, de la Formation des Cadres et de laRecherche Scientifique (MENESFCRS) rapport de synthèse du plan d'urgence 2008-2009.

32. S. Morlaix \& B. Suchaut, Analyse de la réussite en première année universitaire : effets des facteurs sociaux, scolaires et cognitifs. Les documents de travail de l'IREDU, 2, 33 (2012)

33. L. Mucchielli, La pédagogie universitaire en question : le point de vue d'étudiants de premier cycle enpsychologie. Recherche \& Formation Année, 29, 15 (1989)

34. P. Parmentier, Quelques réaction au texte «L'échec à l'université : Comprendre pour mieux prévenir » L'échec à l'université en Communauté française de Belgique. Les Cahiers de recherche en éducation et formation, 39, juin (2005)

35. S. M. Raymond, Effective and ineffective university teaching from the students' and faculty's perspectives: Matched or mismatched expectations? These de doctorat. 
University d'Exeter, UK (2008)

36. J.C. Roda, La carpe et le lapin ou le difficile mariage des universités et des bibliothèques. Bulletin desBibliothèques de France, 41, 2 (1996)

37. M. Romainville. L'échec dans l'université de masse. Paris: L'Harmattan (2000)

38. J. Schmitz, M. Frenay, S. Neuville, G. Boudrenghien, B. Noël, V. Wertz \& J. Eccles, Étude de trois facteurs clés pour comprendre la réussite et la persévérance à l'université. Revue Française de Pédagogie, 172, 18 (2010)

39. M. Trow, Problems in the Transition from Elite to Mass Higher Education. Carnegie Commission onHigher Education, 57p (1973). 


\section{Annexe 1:}

Questionnaire : Perceptions de l'enseignant efficace et de l'enseignement efficace

\section{Perception de l'enseignant efficace}

\begin{tabular}{|c|c|c|c|c|c|}
\hline & & 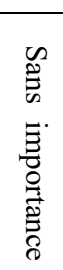 & 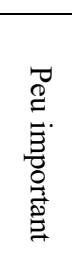 & 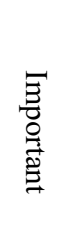 & 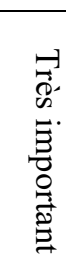 \\
\hline 1 & Patient & & & & \\
\hline 2 & Expérience en enseignement & & & & \\
\hline 3 & Bon communicateur & & & & \\
\hline 4 & Accessible & & & & \\
\hline 5 & Enthousiaste & & & & \\
\hline 6 & Volontaire & & & & \\
\hline 7 & Bon écouteur & & & & \\
\hline 8 & Possède le sens de l'humour & & & & \\
\hline 9 & Expert dans son domaine & & & & \\
\hline 10 & Gentil & & & & \\
\hline 11 & Bien présenté & & & & \\
\hline 12 & Respecte ses étudiants & & & & \\
\hline 13 & Utilise les nouvelles technologies & & & & \\
\hline 14 & Flexible & & & & \\
\hline 15 & Compréhensif & & & & \\
\hline 16 & Confiant en soi & & & & \\
\hline 17 & Approchable & & & & \\
\hline 18 & Dévoué dans son travail & & & & \\
\hline 19 & Dynamique & & & & \\
\hline 20 & Sérieux & & & & \\
\hline 21 & Modeste & & & & \\
\hline 22 & Assidu & & & & \\
\hline
\end{tabular}




\section{CIFEM'2020}

\begin{tabular}{|r|r|l|l|l|l|}
\hline 23 & Explique bien & & & & \\
\hline 24 & Instruit & & & & \\
\hline 25 & Ponctuel & & & & \\
\hline 26 & Sympathique & & & & \\
\hline 27 & Forte personnalité & & & & \\
\hline 28 & Souriant & & & & \\
\hline 29 & Poli & & & & \\
\hline 30 & Modèle pour les étudiants & & & & \\
\hline
\end{tabular}

Perception de l'enseignement efficace

\begin{tabular}{|c|c|c|c|c|c|}
\hline & & 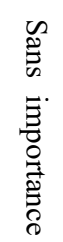 & 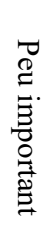 & 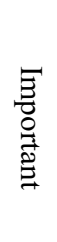 & 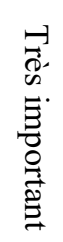 \\
\hline 1 & Implication des étudiants dans le cours & & & & \\
\hline 2 & Maitrise de la matière à enseigner & & & & \\
\hline 3 & $\begin{array}{l}\text { Transmission des connaissances de manière facilitant la } \\
\text { compréhension }\end{array}$ & & & & \\
\hline 4 & Motivation des étudiants et stimulation de leur intérêt & & & & \\
\hline 5 & $\begin{array}{l}\text { L'enseignant prête attention aux besoins (académiques } \\
\text { et personnels) de ses étudiants }\end{array}$ & & & & \\
\hline 6 & Variation de méthodes d'enseignement & & & & \\
\hline 7 & $\begin{array}{l}\text { Développement chez les étudiants du talent de réflexion } \\
\text { et d'analyse }\end{array}$ & & & & \\
\hline 8 & $\begin{array}{l}\text { Développement chez les étudiants de l'esprit critique et } \\
\text { de synthèse }\end{array}$ & & & & \\
\hline 9 & Intégration de support facilitant l'apprentissage & & & & \\
\hline 10 & $\begin{array}{l}\text { Intégration de la technologie favorisant l'amélioration } \\
\text { de la qualité de l'enseignement } \\
\end{array}$ & & & & \\
\hline 11 & $\begin{array}{c}\text { Encouragement des étudiants à participer aux } \\
\text { discussions et à échangerleurs idées }\end{array}$ & & & & \\
\hline 12 & L'évaluation doit être cohérente avec le contenu enseigné & & & & \\
\hline 13 & Pratique de contrôles continus & & & & \\
\hline 14 & Le cours est bien préparé et soigneusement expliqué & & & & \\
\hline 15 & $\begin{array}{l}\text { le contenu du cours doit être cohérent avec le contenu du } \\
\text { TP }\end{array}$ & & & & \\
\hline 16 & $\begin{array}{l}\text { Le contenu du cours est adapté au progrès de la recherche } \\
\text { en la matière }\end{array}$ & & & & \\
\hline 17 & la correction des examens se fait avec responsabilité & & & & \\
\hline
\end{tabular}




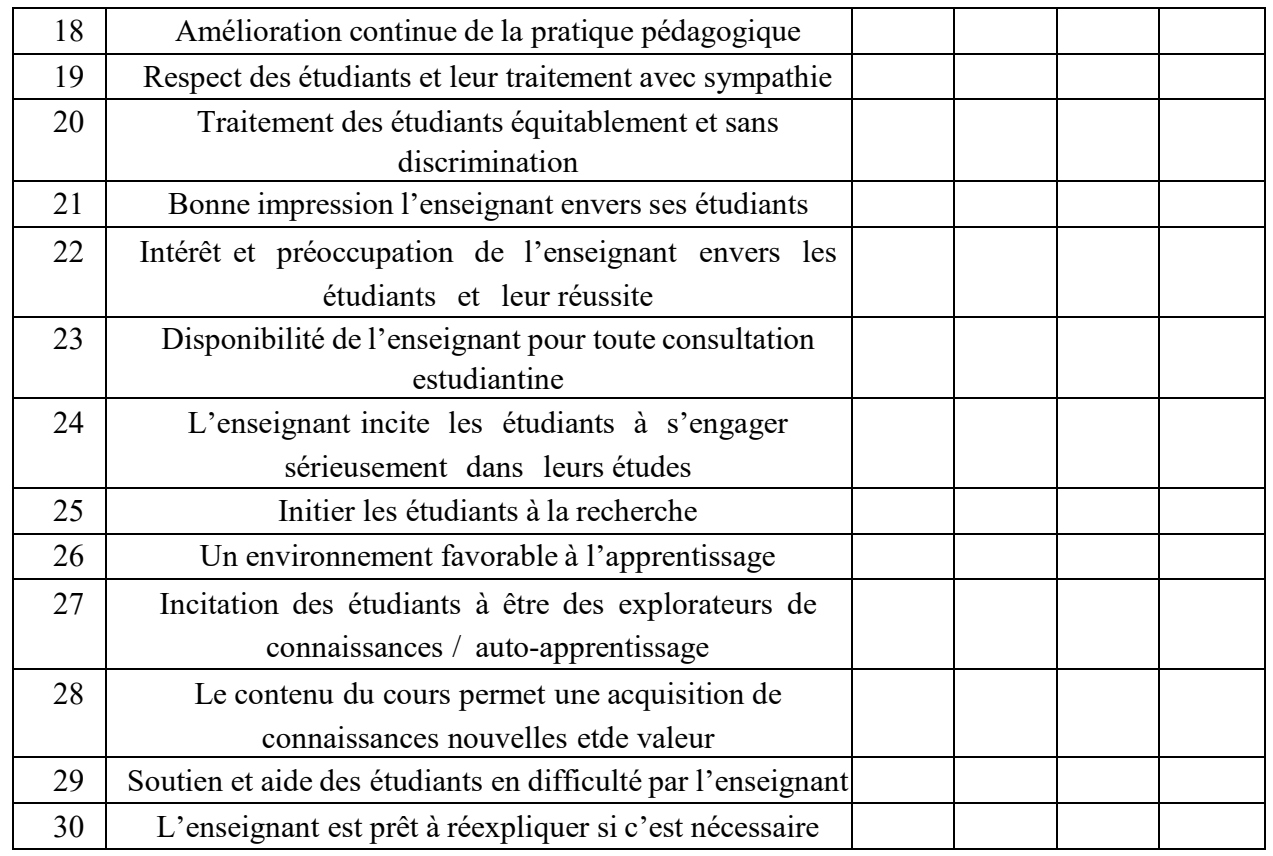

